\title{
Optimizing Municipal Heating Pipeline Confined Space Ventilation by Scale Model Test
}

\author{
Yan Liu, Chunli Yang, Cong Tan, Dongliang Liu \\ Occupational Hazards Control Technology Center, Beijing Municipal Institute of Labour Protection, \\ Beijing 100054, China
}

\section{市政供热有限空间通风优化缩尺模型试验研究}

\author{
刘艳, 杨春丽, 谭聪, 刘东亮
}

北京市劳动保护科学研究所职业危害控制技术中心, 北京市 100054 , 中国

\begin{abstract}
In order to provide effective guide for on-site ventilation of heating pipeline confined space and ensure safety of the workers, the influence of the vents position, the vents numbers, the ventilation air volume and the mixed ventilation on the ventilation effect are studied by scale model test. According to compare the recovery time of carbon dioxide, ventilation effect of each scheme is analyzed. The results show that carbon dioxide recovery time is shorter and the ventilation effect is better when air is pressured into through the middle vent and free outflow through vents at both ends, and when air is pressured into through vents at one end and pumped out through vent at the other end. When the ventilation volume and the vent position are determined, the excessive air vent number will not shorten the time required for the ventilation. The increase of ventilation air volume will reduce the recovery time of carbon dioxide to a certain extent, but there is no linear relationship between the two parameters. When the air volume increases to a certain value, reduction of ventilation time is very small.
\end{abstract}

Key words: confined space; scale model; test; ventilation effect; carbon dioxide
摘要

为有效指导市政供热有限空间机械通风, 保障作业人员安全, 采用缩尺模型实验的方法, 研究通风口位置、通风口数量、通风风量以及 混合式通风对通风效果的影响, 通过对比二氧 化碳的恢复时间, 分析各通风方式的优劣。研 究结果表明采用中部压入两端出风, 或采用一 端压入另一端抽出的通风方式时, 二氧化碳恢 复正常所需时间较短, 通风效果较好; 在通风 风量与通风口位置确定的情况下, 过多增加通 风口数量不会缩短整体通风所需的时间; 增加 通风风量, 在一定程度上会使模型内二氧化碳 含量恢复正常的时间减少, 但是通风风量的增 加与通风时间的减小之间并非线性关系, 风量 增加到一定数值以上时, 通风时间降低幅度较 小。

关键词: 有限空间; 缩尺模型; 实验; 通风效 果; 二氧化碳

\section{1. 引言}

市政地下有限空间不同于地面设施, 由于 其长期处于封闭或半封闭状态, 且出入口有限, 自然通风不良, 极易造成有毒有害气体的积聚, 以及氧气浓度偏低, 工人在其内部作业存在一 定的安全隐患 ${ }^{[1,2]}$ 。近年来, 随着我国城市化 进程的加快，水、电、气、热等市政地下管线 大量敷设, 伴随而来的是市政地下有限空间作 业频次和从业人员数量急剧增加, 市政地下有 限空间作业事故屡有发生, 据统计, 北京市 2006-2015 年间共发生 43 起市政地下有限空 
间作业事故, 占有限空间作业事故的 $84 \%{ }^{[3]}$ 。 为保证有限空间作业人员安全, 国内外相关标 准中均明确规定, 进入有限空间作业前必须先 通风，待有限空间内氧气、有毒有害气体、易 燃易爆气体的检测值均合格后方可进入其内 部作业 ${ }^{[4-6]}$ 。然而在实际作业中, 由于缺乏有 效的技术支撑, 现场机械通风具有一定的盲目 性, 这些不仅影响作业效率, 而且还可能因管 沟内通风不彻底而留下安全隐患。

目前, 国内外针对有限空间通风方面研究 较少, 仅个别学者做了少量研究, Richard ${ }^{[7]}$ 通过研究认为在有限空间通风过程中, 压入式 通风比抽出式通风氧含量恢复快, 且氧含量随 时间呈指数函数变化。 $\mathrm{J} \mathrm{Zhao}{ }^{[8]}$ 采用数值模拟 的方法研究了机械通风过程中化粪池内硫化 氢、二氧化碳、氧气等气体的变化规律, 认为 化粪池内充满任何一种有毒有害气体 (硫化氢、 二氧化碳等) 时, 氧气的恢复时间差别很小。 EP Pesce ${ }^{[9]}$ 通过实验研究了化粪池通风过程中 硫化氢气体的变化规律, 并根据实验结果确定 了最优通风方案; Liu Yan ${ }^{[10]}$ 采用 CFD 技术对 供热有限空间通风过程进行模拟仿真, 研究通 风过程中氧含量、二氧化碳含量、温度等动态 变化规律。这些研究为有限空间通风风量、通 风时间等参数的设定提供一定参考, 但是目前 市政机械有限空间通风方式的选择、通风时间 的长短依然不太明确, 相关研究还有待于深入。 本文以市政供热有限空间为研究对象, 采 用相似实验的研究方法, 考虑通风口位置、数 量、通风风量、通风时间等情况, 研究不同参 数条件对通风效果的影响, 以期为相关标准法 规的制定、现场实际作业等提供技术支撑。

\section{2. 实验装置和实验方案}

\section{1 实验装置}

依据北京市某一供热有限空间的结构尺 寸, 按照 $1: 5$ 的缩尺比例设计并加工试验装置, 装置包括检查室和管沟两部分, 两条管沟位于 检查室的两侧, 为了加工方便, 半拱形管沟简 化为方形管沟。检查室尺寸为 $1.2 \mathrm{~m} \times 1.2 \mathrm{~m} \times 1.2 \mathrm{~m}$; 两侧管沟断面尺寸均为 $0.6 \mathrm{~m} \times 0.4 \mathrm{~m}$, 长均为 $10.5 \mathrm{~m}$ 。试验装置布置有 通风口和监测点, 布置示意图如图 1 ; 其中, 通风口共有 5 个, 直径均为 $0.15 \mathrm{~m}$, 分别如图 1
中 A、B、C、D和E, A和E通风口距检查室中心 $10.7 \mathrm{~m}, \mathrm{~B}$ 和D通风口距检查室中心 $5.6 \mathrm{~m}$, C通 风口位于检查室上部。监测点共布置 5 个, 分 别如图1中的 $1 、 2 、 3 、 4 、 5$ 位置, 其中 1 号和 4 号监测点距检查室中心 $8.1 \mathrm{~m}, 2$ 号和 3 监测点距 检查室中心 $3.1 \mathrm{~m}, 5$ 号监测点位于检查室内, 距离检查室顶端 $0.6 \mathrm{~m}$; 在监测点位置布置气体 传感器, 用以监测气体含量的变化。实验装置 示意图如图2所示。

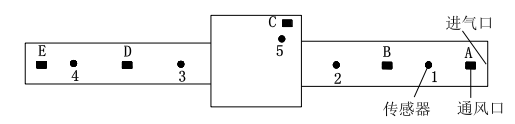

图 1 通风口和监测点布置示意图

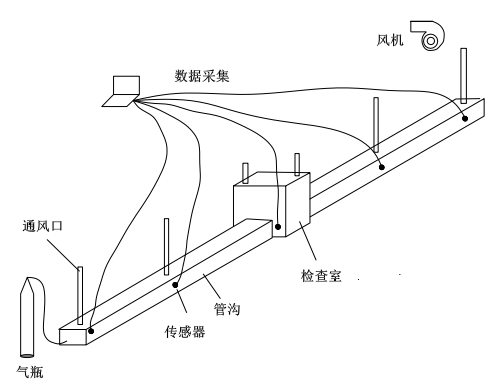

图 2 实验装置示意图

\section{2 实验方案设计}

由于难以实现在实验装置内使氧含量减 少, 采用在实验装置内通入一定量的二氧化碳, 在通风过程中通过监测二氧含碳含量的变化 评估通风效果的优劣。在实验过程中, 将纯度 为 $99.9 \%$ 含量的二氧化碳气体以 $5 \mathrm{~L} / \mathrm{min}$ 的速率 通过检查室中间通风口通入至实验装置中, 通 气时间为 32 分钟, 通气过程中各个通风口均封 闭。通气结束后, 开始通风实验。

实验考虑进风口位置、出风数量, 进风风 量和混合式通风共 4 类因素, 共设计 16 组通风 实验方案, 如表 1 -表 4 所示。在实验过程中采 用三种型号的风机, 分别为 1 号风机, 2 号风机 和 3 号风机。其中 1 号风机风量为 $80 \mathrm{~m}^{3} / \mathrm{h}, 2$ 号 风机为可调风量风机, 两档风量分别为 $120 \mathrm{~m}^{3} / \mathrm{h}$ 和 $160 \mathrm{~m}^{3} / \mathrm{h}, 3$ 号风机也为可调风量风机, 两档风量分别为 $200 \mathrm{~m}^{3} / \mathrm{h}$ 和 $240 \mathrm{~m}^{3} / \mathrm{h}$ 。除表 3 方案 外, 其它方案采用 2 号风机, 通风风量均为 $160 \mathrm{~m}^{3} / \mathrm{h}$, 且表中未标明的通风口即为关闭状 态, 通风过程中进气口为关闭状态。 
表 1 进风口位置对通风效果的影响

\begin{tabular}{|c|c|c|c|c|}
\hline 方案号 & & 出风口编号 & \multicolumn{2}{|c|}{ 开启进风口编号 } \\
\hline $1-1$ & & \multicolumn{2}{|c|}{ A E } & B \\
\hline $1-2$ & & \multicolumn{2}{|c|}{$A E$} & $\mathrm{C}$ \\
\hline $1-3$ & & \multicolumn{2}{|c|}{$\mathrm{C} \mathrm{E}$} & A \\
\hline \multicolumn{5}{|c|}{ 表 2 出风口数量对通风效果的影响 } \\
\hline 方案 & & 进风口 & 出风口 & 出风口 \\
\hline 号 & & 编号 & 数量 & 编号 \\
\hline $2-1$ & & $\mathrm{C}$ & 2 & A E \\
\hline $2-2$ & & $\mathrm{C}$ & 3 & A B E \\
\hline $2-3$ & & $\mathrm{C}$ & 3 & A D E \\
\hline $2-4$ & & $\mathrm{C}$ & 4 & A B D E \\
\hline \multicolumn{5}{|c|}{ 表 3 进风风量对通风效果的影响 } \\
\hline \multicolumn{2}{|c|}{ 方案号 } & $\begin{array}{c}\text { 进风 } \\
\text { 口编 } \\
\text { 号 }\end{array}$ & $\begin{array}{l}\text { 出风口 } \\
\text { 编号 }\end{array}$ & $\begin{array}{l}\text { 通风风 } \\
\text { 量 }\left(\mathrm{m}^{3} / \mathrm{h}\right)\end{array}$ \\
\hline \multicolumn{2}{|c|}{$3-1$} & $\mathrm{C}$ & A E & 80 \\
\hline \multicolumn{2}{|c|}{$3-2$} & $\mathrm{C}$ & A E & 120 \\
\hline \multicolumn{2}{|c|}{$3-3$} & $\mathrm{C}$ & A E & 160 \\
\hline \multicolumn{2}{|c|}{$3-4$} & $\mathrm{C}$ & A E & 200 \\
\hline \multicolumn{2}{|c|}{$3-5$} & $\mathrm{C}$ & A E & 240 \\
\hline \multicolumn{5}{|c|}{ 表 4 混合式通风对通风效果的影响 } \\
\hline $\begin{array}{l}\text { 方 } \\
\text { 案 } \\
\text { 号 }\end{array}$ & $\begin{array}{l}\text { 进 } \\
\text { 风 } \\
\text { 口 }\end{array}$ & \multicolumn{2}{|c|}{ 出风口编号 } & $\begin{array}{c}\text { 排风风 } \\
\text { 量 }\end{array}$ \\
\hline $4-1$ & A & \multicolumn{2}{|c|}{$\begin{array}{c}\text { E（未设机械排 } \\
\text { 风） }\end{array}$} & - \\
\hline $4-2$ & A & \multicolumn{2}{|c|}{$\begin{array}{c}\mathrm{E} \text { (E 处设机械排 } \\
\text { 风) }\end{array}$} & $80 \mathrm{~m}^{3} / \mathrm{h}$ \\
\hline $4-3$ & $\mathrm{C}$ & \multicolumn{2}{|c|}{$\begin{array}{c}\text { A E（未设机械排 } \\
\text { 风） }\end{array}$} & - \\
\hline $4-4$ & C & \multicolumn{2}{|c|}{ 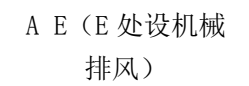 } & $80 \mathrm{~m}^{3} / \mathrm{h}$ \\
\hline
\end{tabular}

\section{3. 实验结果分析}

\section{1 进风口位置对通风效果的影响}

为研究进风口位置对通风效果影响, 按照 表1中方案进行通风实验, 不同方案结果如图 3-图5所示。

由图3-图5可知:

（1）当按照方案 1- 1 的设置进行通风时, 各监测点二氧化碳含量降至最低且保持稳定 需要的时间为 9 分钟; 当按照方案 $1-2$ 的设置进 行通风时, 各个监测点二氧化碳含量降至最低 且保持稳定需要的时间为 7 分钟; 当按照方案 1-3的设置进行通风时, 各监测点二氧化碳含 量降至最低且保持稳定需要的时间为 25 分钟。 三种通风方案下二氧化碳含量降至最低且保 持稳定所需时间排列为方案 1-3>方案1-1>方
案1-2。因此, 针对本实验模型, 将风机放置 在管沟中部的检查室上方, 所需通风时间最短, 通风效果最好。

（2）对于管沟内的监测点, 当风机按照 方案 1- 1 和方案 $1-3$ 的设置进行通风时, 1 号监 测位二氧化碳含量降到最低所需的时间最短, 4 号监测位二氧化碳含量降到最低所需的时间 最长; 当风机按照方案 1-2的设置进行通风时, 5 号监测位二氧化碳含量降到最低所需的时间 最短, 4 号监测位二氧化碳含量降到最低所需 的时间最长。

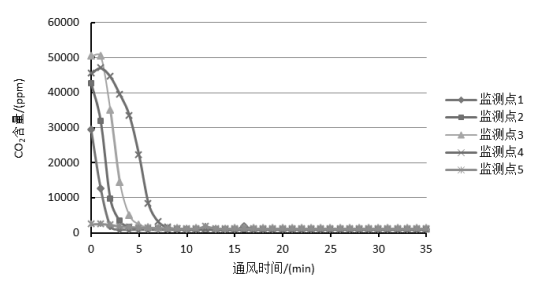

图3 方案1-1通风时各监测点二氧化碳含量随时间 变化曲线

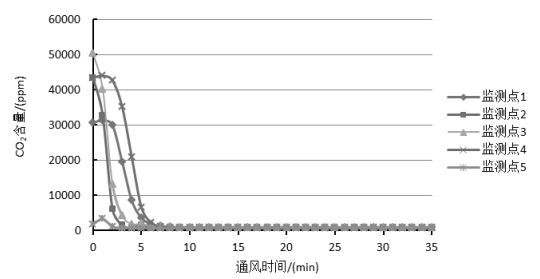

图4 方案1-2通风时各监测点二氧化碳含量随时间 变化曲线

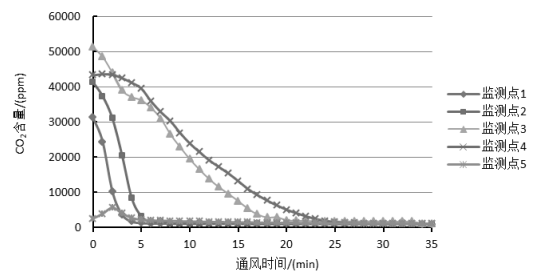

图 5 方案 1-3 通风时各监测点二氧化碳含量随时间 变化曲线

\section{2 进风口数量和位置对通风效果影响}

为研究通风口数量和位置对通风效果的 影响, 按照表2 方案进行通风实验, 由于方案 2-1和方案1-2相同, 直接采用方案1-2的结果。 不同方案的结果如图6-图9所示。 


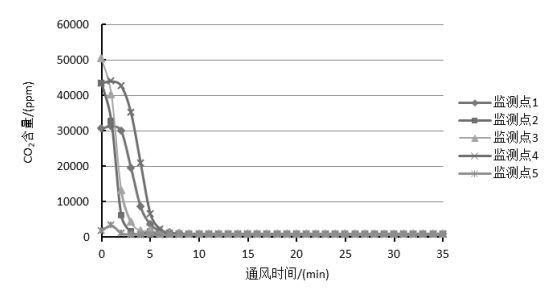

图6 方案2-1通风时各监测点二氧化碳含量随时间 变化曲线

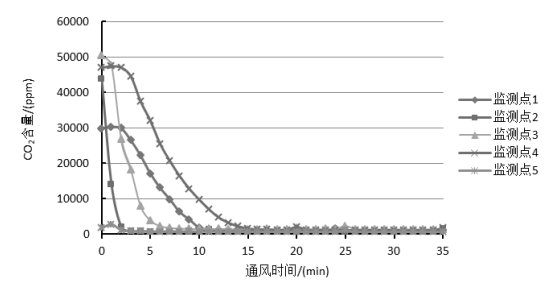

图7 方案2-2通风时各监测点二氧化碳含量随时间 变化曲线

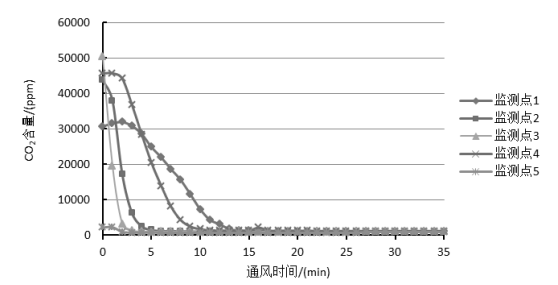

图8 方案2-3通风时各监测点二氧化碳含量随时间 变化曲线

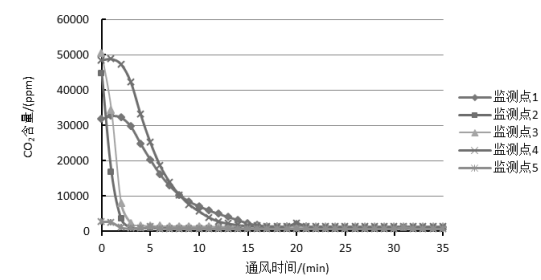

图 9 方案 2-4 通风时各监测点二氧化碳含量随时间 变化曲线

由图6-图9可知:

(1) 当开启 $\mathrm{A}$ 号和 $\mathrm{E}$ 两个通风口时, 各个 监测点二氧化碳含量降至最低且保持稳定需 要的时间为7分钟; 当开启三个通风口时, 开 $A 、 B$ 和E时各监测点二氧化碳含量降至最低且 保持稳定需要16分钟, 开启A、D和E时需要 14 分钟; 当开启四个通风口即通风口全开时, 各 个监测点二氧化碳含量降至最低且保持稳定 需要的时间为 17 分钟。按照通风所需时间排列,
各个方案由多到少为方案 2-4 $>$ 方案 2-2 $>$ 方案 2-3>方案2-1, 即通风口数量越多, 各个监测 二氧化碳恢复到正常值的时间越长。

(2) 对于每一通风方案, 距离风机较近 的监测点 2 和 3 二氧化碳恢复到正常值的时间 比距离风机较远的监测点 1 和 4 号所需时间短, 且不同通风方案下监测点 $2 、 3$ 二氧化碳含量恢 复到正常值的时间不同，按照所需时间由多到 少排列为方案2-2>方案2-3>方案2-1>方案2-4, 因此可看出增加通风口数量有利于缩短检查 室内和距离检查室较近的管沟内二氧化碳含 量恢复正常值的时间, 但是对于管沟内距离进 风口较远的位置, 增加通风口数量会增加该位 置二氧化碳含量整体恢复到正常的时间。

\section{3 通风风量对通风效果影响}

为研究通风风量对通风效果的影响, 按照 表3中方案进行通风实验, 不同方案结果如图 10-图14所示。

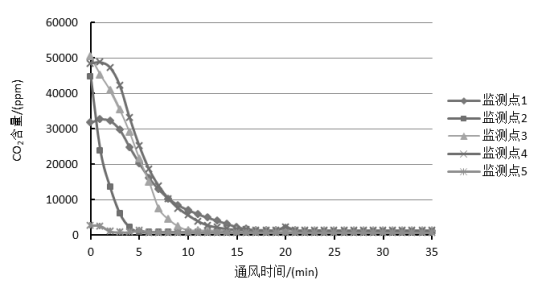

图10 方案3-1时各监测点二氧化碳含量随时间变 化曲线

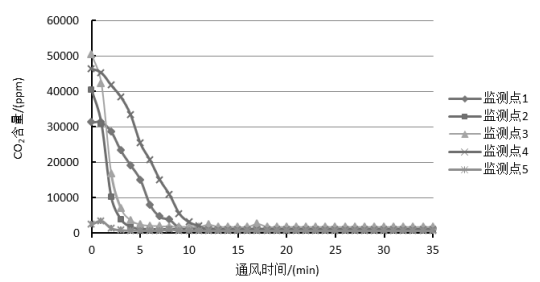

图11 方案3-2时各监测点二氧化碳含量随时间变 化曲线

由图 10-14 可知: 当风机风量分别为 $80 \mathrm{~m}^{3} / \mathrm{h} 、 120 \mathrm{~m}^{3} / \mathrm{h} 、 160 \mathrm{~m}^{3} / \mathrm{h} 、 200 \mathrm{~m}^{3} / \mathrm{h}$ 和 $240 \mathrm{~m}^{3} / \mathrm{h}$ 时, 监测点二氧化碳含量恢复正常所需时间分 别为 16 分钟、 12 分钟、 7 分钟、 6 分钟和 5 分钟, 即随着通风风量的增加, 管沟模型内二氧化碳 含量降至稳定值所需时间逐渐减小。风量增加 数值与通风时间减少值之间关系如图15所示, 
图中风量的增加值和通风时间减少值均是与 方案3-1相比, 从图中可以看出通风风量的增 加与通风时间的减小之间并非线性关系, 当风 量增加到 $200 \mathrm{~m}^{3} / \mathrm{h}$ 以上时, 二氧化碳含量降低 至最低所需时间并没未显著减少。分析原因可 能是进风风量过大而使管沟内部湍流程度加 强, 导致局部区域出现通风死角。

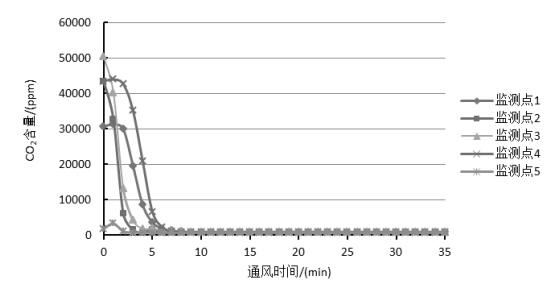

图12 方案3-3时各监测点二氧化碳含量随时间变化 曲线

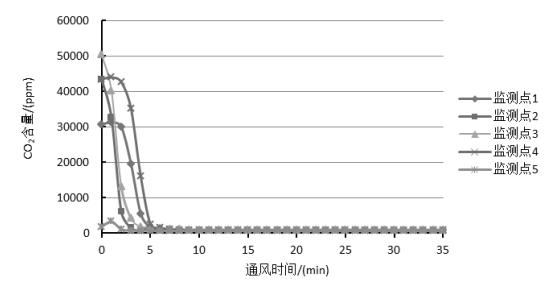

图13 方案3-4时各监测点二氧化碳含量随时间变化 曲线

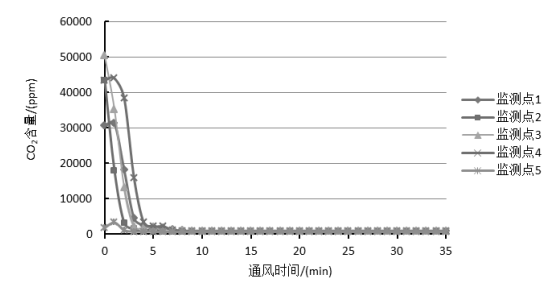

图14 方案3-5 时各监测点二氧化碳含量随时间变化 曲线

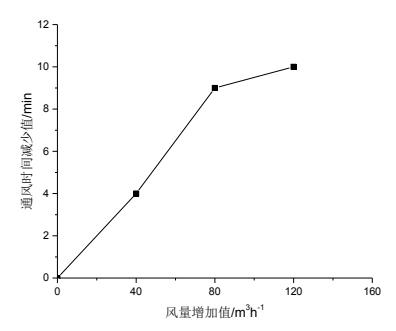

图15 风量增加与通风时间减小之间关系图

\section{4 混合式通风对通风效果影响}

为了探究混合式通风对通风效果的影响, 按照表4中方案进行通风实验, 不同方案结果 如图16-图19所示。

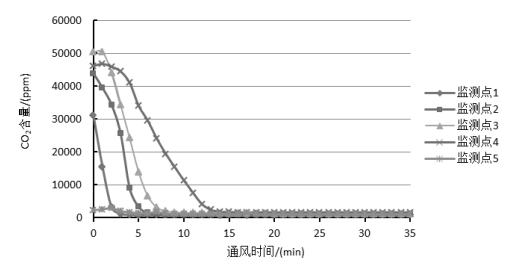

图 16 方案 4-1 时各监测点二氧化碳含量随时间变 化曲线

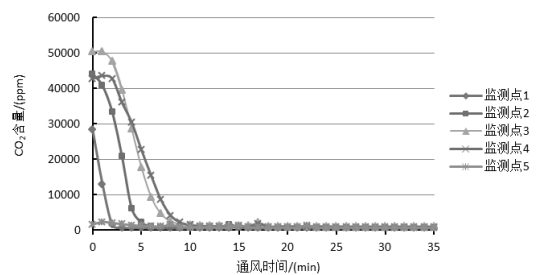

图 17 方案 4-2 时各监测点二氧化碳含量随时间变 化曲线

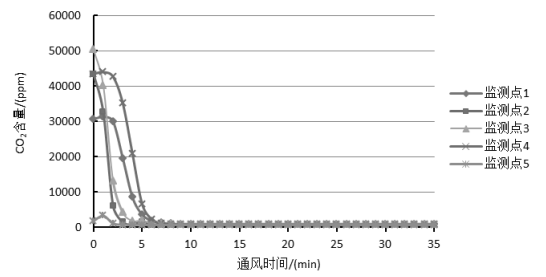

图 18 方案 4-3 通风时各监测点二氧化碳含量随时 间变化曲线

由图16-图19可知: 在不设混合式通风的 情况下, A通风口进风, $\mathrm{C}$ 号通风口出风时, 二 氧化碳含量恢复正常需要15分钟; 在设置机械 排风后, 二氧化碳含量恢复正常所需时间为 10 分钟; 在不设置机械排风时, C为通风口进风, $\mathrm{A}$ 号 $\mathrm{E}$ 号通风口出风时, 二氧化碳含量恢复正常 需要 7 分钟; 在设置机械排风后, 二氧化碳含 量恢复正常所需时间为 5 分钟。从对比可以看 出, 采用混合式通风可缩短通风时间。

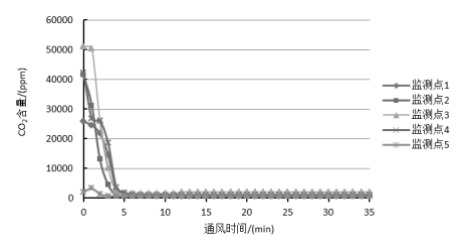

图 19 方案 4-4 时各监测点二氧化碳含量随时间变 化曲线 


\section{4. 结论}

(1) 对于本模型, 即检查室两端为双侧 通行沟的情况, 在将风机放置在检查室顶部进 风，管沟两端检查室排风的通风方式下，二氧 化碳含量恢复正常所需的时间最短, 通风效果 最好, 其他通风口位置方案下二氧化碳含量恢 复正常所需时间相对较长。

（2）在通风风量与通风口位置确定的情 况下, 增加通风口数量能够使风机附近的二氧 化碳含量快速恢复正常, 但是对于距离风机较 远的管沟内, 其内部二氧化碳含量恢复正常的 时间会相对较长, 即过多增加通风口数量不会 缩短整体通风所需的时间。

（3）增加通风风量, 在一定程度上会使 模型内二氧化碳含量恢复正常的时间减少, 但 是通风风量的增加与通风时间的减小之间并 非线性关系, 风量增加到一定数值以上时, 通 风时间降低幅度较小。

(4) 将普通通风方式换为混合式通风, 能够加快模型内二氧化碳的排出, 缩短管沟内 气体含量恢复正常所需的时间。

\section{Acknowledgements}

This study was supported by Beijing Academy of Science and Technology Building of Innovative Team Plan (No. IG201702C1) and Natural Science Foundation of Beijing Municipality (No. 8152014).

\section{致谢}

本研究得到了北京市科学技术研究院创新团 队计划课题 (IG201702C1) 和北京市自然科学 基金（8152014）的资助。

\section{参考文献}

[1] Silverberg P. Safety Health in Confined Space. CRC press, Flordia, 1998.

[2] 刘艳, 秦妍. 有限空间作业安全. 团结出 版社, 北京, 2016.

[3] 刘艳, 杨春丽. 有限空间作业事故特征及 其原因分析. 中国安全科学学报,2017, 27(3): 141-145.
[4] 国家安全监管总局. 工贸企业有限空间 作业安全管理与监督暂行规定, 2013.

[5] 29 CFR 1910,146. Permit-Required Confined Spaces, 1993.

[6] AS/NZS 2865, Safe working in a confined space, 2001.

[7] Richard P G, Ravi N, Muzaffer E. Ventilation to eliminate oxygen deficiency in a confined space part I: a cubical model. Applied Industrial Hygiene,1989, 4(4): 1-11.

[8] Zhao J, Manbeck H B, Murphy D J. Computational fluid dynamics simulations of gas evacuation and $\mathrm{O}_{2}$ recovery times for fan-ventilated confined-space manure pits. Studies in Foreign Education, 2008, 51(6): 2135-2149.

[9] Pesce E P, Zhao J, Manbeck H B, Murphy D J. Screening ventilation strategies for confined-space manure storage facilities. Journal of Agricultural Safety \& Health, 2008, 14(3):283-308.

[10] Liu Y, Tan C, Liu D L. CFD analysis of oxygen and carbon dioxide recovery during ventilation in municipal confined space. Journal of Risk Analysis and Crisis Response, 2016, 6(4): 178-185. 